\title{
Overview on Electric Activity and Collective Behaviors of Diffusion System

\author{
${ }^{1}$ College of Electrical Engineering, Northwest University for Nationalities, China \\ ${ }^{2}$ College of Journalism and Communication, Northwest University for Nationalities, China \\ azhoujianfrank@126.com, bcaining91@tsinghua.org.cn, ckonglingjin928@126.com
} \\ Jian Zhou ${ }^{1, \text { a }}$, Ning Cai ${ }^{1, b^{*}}$ and Lingjin Kong ${ }^{2, c}$ \\ *The corresponding author
}

\section{Keywords: Diffusion system; Electric activity; Collective behaviors}

\begin{abstract}
Heterogeneity exerts a repulsive force on the approaching spiral tip, and the attractive force prevents the spiral tip from unpinning from the heterogeneity once the tip has anchored electromagnetic radiation on electrical activities of neuron, energy consumption in neurons are presented. Autapse is an unusual type of synapse generated by a neuron on itself. The effect of autapse connected to a neuron is often described by using a self-feedback forcing current in a close loop, and the electric activities of neuron can be regulated by the autapse greatly. Generally, positive feedback in the autapse can excite the quiescent neuron while negative feedback often calms down the excitable neuron.
\end{abstract}

\section{Introduction}

In a neuronal system, information can be transmitted between neurons via synapses, and the electrical activity of a given neuron can be changed by other neurons that synapse onto it. Furthermore, the electrical behavior of some neurons can be heavily affected by the presence of autapses, which is an unusual type of synapse generated by a neuron on itself [1].Some researchers suggested that the effect of autapse on electric activities of neuron can be described by inputting additive loop current with time delay similar to the external forcing current on the neuron [2], as a results, a positive feedback with time delay can excite the quiescent neuron to become a continuous oscillator and generate different types of electric activities such as spiking, bursting and chaotic states. Particularly, Wang et al. [3] investigated the transition of activities in Hindmarsh-Rose neurons that had three different types of autapses by using bifurcation analysis and calculating distribution of information entropy. In fact, time delayed-feedback (negative feedback) can suppress the chaotic state in oscillator [4] and spiral wave in the media [4, 5]. For spiking or bursting states in neurons, autapses with negative time delayed-feedback can stabilize the neuron to step into quiescent state, and hence it is interesting to investigate the case when autapses are considered in a local area in the network (only a fraction of neurons are connected to autapse not all neurons are connected to autapses). Some previous results have confirmed that continuous pulse or target wave or spiral wave can be developed in the network when some autapses connected the neurons are in positive time delayed-feedback type [6]. As mentioned in Refs, autaptic modulations can shape the bursting behavior of biological neurons, and they confirmed that synaptic delays have no obvious effects in the case of autaptic-excitation, while a subtle effect of synaptic delays was observed in the case of autaptic-inhibition. As mentioned above, the response of neuronal activity is much dependent on the feedback type (negative or positive) in autapse, and the transition of dynamical properties could be interesting. For an isolated neuron, a switch selection between positive and negative feedback in autapse make neuron become excitable or quiescent. As a result, transition of spatial pattern occurs when collective electrical activities of neuronal network are detected. Therefore, the development and transition of electric activities of neurons in network can be more reliable to give useful clues to understand the neuronal activities and response to external forcing. We argue that negative time delayed-feedback in autapse connected to some neurons in a 
local area of the network may generate 'defects' in the network, and ordered wave can also emit from these defects to regulate the collective behaviors of neurons. For simplicity, the Hindmarsh-Rose neuron model is used to describe the local kinetics of nodes in the neuronal network with nearest-neighbor connection.

The neuronal system contains a large number of neurons; the electric activities of each neuron can show quiescent, periodical and/or chaotic state under appropriate intrinsic parameter selection and external forcing. The development of nonlinear science paves a reliable way to understand the transition of neuronal activities in neurons by using some reliable neuron models [7].The dynamical properties of electric activities, stochastic resonance, coherence and synchronization of neurons have been investigated extensively [8,9].Furthermore, the collective behaviors of neuronal network have also been investigated by detecting the spatiotemporal pattern selection, transition of spatial distribution [10], such as pulse wave [11] in a ring network, target wave [12], spiral wave [11-13,14-15] in a two-dimensional array with different topological structures. In fact, in realistic neuronal system, the effect of other factors should be considered, for example, the action potential is also dependent on the gliocyte and it is interesting to study the cooperative behaviors of neuron and astrocyte Channel noise and external noise also plays an important role in regulating the electrical activity of a single neuron, or neurons in a network, for example, Ref. [16] investigated the transition of spiral wave in neuronal networks induced by channel noise. The channel noise can be altered when the conductance of ion channels is changed and this can result in a loss of stable behavior due to blocking in ion channel [17].One may then ask, why investigate spiral waves in neuronal networks? In fact, some experimental results have confirmed that spiral wave can emerge in the cortex of brain, and these spiral waves seems to regulate the collective behaviors of neurons as a pacemaker, as a result Refs presented detailed discussion about the potential formation mechanism for spiral wave (one arm or multi-armed type), and transition of ordered wave induced by changeable connection probability in the network, and their previous results claimed that ion channel blocking in some neurons may account for the emergence of spiral wave in the network.

\section{Preliminaries}

Parameter collapse-induced instability of neuronal network is investigated in a two-dimensional square array with nearest-neighbor connection, and the local kinetics is described by neuron model. Regular spatial distribution is generated by developing target waves under external forcing current with diversity in the network of ML neurons [18], or producing local heterogeneity in parameter in the network of Hindmarsh-Rose neuron. The sampled time series for membrane potentials and correlation functions from three nodes are detected to analyze transition in electrical activities, so that collapse in network could be predicated before final collapse in regularity of network. It finds that the regular distribution in network could be destroyed completely with increasing the collapse area beyond certain threshold. The collapse in different parameters causes different deformation degrees for the network, and collapse in some critical parameters can induce rapid breakup of target wave in the network. The network distribution can also keep its regularity and robustness to collapse in certain parameter. In fact, the collapse of network cannot be predicated and prevented when the collapse in parameter is diffused with high speed (smaller diffusion period).Sometimes, the collapse could be predicated by analyzing the correlation functions for more monitored nodes if possible. For the network of neurons including ion channels, its mechanism could be that drug absorption and diffusion make more and more ion channels be poisoned and thus the nervous system encounters breakdown finally due to diffusive effect. For ecological system such as forest, fire or earthquake, even infectious disease in one region can ruin the balance of the ecological system, therefore, it is important to monitor some nodes in this area so that occurrence of disaster could be predicated as soon as possible.

\section{Dynamics of Collective Behaviors of Network of Neurons}

Dynamical Analysis. The four-variable Hodgkin-Huxley $(\mathrm{HH})$ neuron model can reproduce the 
properties of electric activities in axon of squid and the stochastic effect of ion channels embedded in the membrane could be estimated. For available dynamical analysis, a three-variable Hindmarsh-Rose (HR) neuron model is developed by simplifying the original HH neuron model. The dynamical equations for HR neuron model are described by

$$
\left\{\begin{array}{l}
\dot{x}=y-a x^{3}+b x^{2}-z+I_{e x t} \\
\dot{z}=r\left[s\left(x-x_{0}\right)-z\right] \\
\dot{y}=c-d x^{2}-y
\end{array}\right.
$$

where the variables $\mathrm{x}, \mathrm{y}, \mathrm{z}$, represent the membrane potential, he transmembrane fast current associated with recovery variable, adaption current for slow variable, respectively. This model can generate quiescent, spiking, bursting even chaotic state by changing the external forcing current carefully. Indeed, bifurcation analysis in interspike interval (ISI) is effective on understanding the modes transition in electrical activities in neuron even with fractional-order dynamical system.Extensive studies confirm that the Morris-Lecar neuron model with fractional-order can present abundant bursting patterns and dynamics by bifurcation analysis. For a detailed bifurcation analysis in the HR model, readers can refer to ref. Interestingly, a modified HR neuron model with four variables and thirteen parameters was presented to generate more complex dynamical behaviors, which is shown as follows:

$$
\left\{\begin{array}{l}
\dot{x}=y-a x^{3}+b x^{2}-z+I_{e x t} \\
\dot{y}=c-d x^{2}-y-e w \\
\dot{z}=r\left[s\left(x-x_{0}\right)-z\right] \\
\dot{\omega}=h[-p \omega+f(y+g)]
\end{array}\right.
$$

Functional Action of Autapse. The biological experiments have confirmed that a specific synapse, which connects to its own soma or dendrite via a loop, is named autapse that can regulate the membrane potential or excitability of media by using time-delayed feedback type The membrane potential of neuron can be regulated by autapse with electrical type and chemical type by imposing additive feedback current with time delay. The electrical and chemical autapse can be described by

$$
\left\{\begin{array}{l}
I_{\text {out_electrical }}=g_{e}[x(t)-x(t-\tau)] \\
I_{\text {out_chemical }}=-\frac{g_{c}\left[x(t)-V_{s y n}\right]}{1+e^{-\lambda[x(t-\tau)-\theta]}}
\end{array}\right.
$$

A positive feedback is helpful to exciting the quiescent neuron while a negative feedback does calm down the excitable neuron completely. We are often puzzled at why neuron can give different responses to the same external forcing, and could give the same response under different external forcing. In our view, the potential mechanism could be associated with the self-adaption function from autapse, which can be realized by selecting appropriate time delay $\tau$ and gain $g$ in the close loop. It also indicates that time delay plays an important role in changing Excitability of neuron.

Open Problems. Based on the known neuron models, the nonlinear analysis could be carried out in available way. However, there are some problems that should be further investigated because these neuronal models are much simplified. In realistic nervous system, the modulation from astrocyte on membrane potential, the distribution of autspse, boundary effect of heterogeneity, even electromagnetic radiation should be considered. Astrocyte cells are traditionally considered as non-excitable support cells of neuron systems because these cells cannot generate action potentials (APs).

Three variable HR neuron model and application of memristor, we proposed a modified neuronal model so that the effect of electromagnetic radiation could be described by introducing additive variable as magnet flux into the original HR neuron model based on the mean field theory, and a new improved neuron model could be described by 


$$
\left\{\begin{array}{l}
\frac{d x}{d t}=y-a x^{3}+b x^{2}-z+I_{e x t}-k_{1} \rho(\varphi) x \\
\frac{d y}{d t}=c-d x^{2}-y \\
\frac{d z}{d t}=r[s(x+1.6)-z] \\
\frac{d \varphi}{d t}=x-k_{2} \varphi \\
\frac{d q(\varphi)}{d t}=\rho(\varphi)
\end{array}\right.
$$

\section{Discussion}

The transition of spatial patterns from regular states to broken states is investigated in the neuronal network with no-flux boundary condition being used. The diffusive collapse in parameters is generated by switching certain parameter to another value, and the size of affected area is increased with time. For the network of neurons embedded ion channels, its background could be that more and more ion channels embedded in the. It is found that the profile of the diffusive damage area is much dependent on the diversity in parameter $a$ even if the damage area is expanded with the same velocity or rating. When the ordered spiral wave is invaded by the diffusive damage area, the wave front is blocked and thus breakup of spiral wave occurs in local area. membranes of neurons are blocked by poisoning and drug diffusion. Firstly, parameter collapse-induced transition of spatial pattern in the neuronal network of ML neurons is investigated; three nodes in the network will be monitored by detecting the sampled time series for membrane potentials. The results illustrate that both spectral rigidity and number variance show a "hiding the head, showing the tail" shape, as found in previous studies. For small L, long-range correlation indexes agree well with the values predicted by the GOE statistic, reflecting the universal behavior of the dynamic brain network.

\section{References}

[1] Jun Ma, Wuyin Jin: International Journal of Modern Physics-B, (2015), Emergence and Robustness of Target Waves in a Neuronal Network.

[2] Jun Ma, Jun Tang: Communications in Nonlinear Science and Numerical Simulation, Detection of Noise Effect on Coupled Neuronal Circuits.

[3] Sinha, Saramaki and Kaski: Phys Rev E, (2007); 76:015101, Emergence of Self-sustained Patterns in Small-world Excitable Media.

[4] Wuyin Jin, Jun Ma and Z.B. Xie: Acta Phys Sin, (2013); 62:240507, Effect of Inhomogeneous Distribution of Ion Channels on Collective Electric Activities of Neurons in a Ring Network.

[5] Jun Ma, C.N. Wang and H.P. Ying: Sci China Phys Mech Astro, (2013); 56:1126-38, Emergence of Target Waves in Neuronal Networks Due to Diverse Forcing Currents.

[6] Jun Ma, L Huang and H.P. Ying: Chin Sci Bull, (2012);57:2094-101, Detecting the Breakup of Spiral Waves in Small-world Networks of Neurons Due to Channel Block.

[7] Jun Ma, Chunni Wang and Ying Wu: Plos One, (2014), Autapse-Induced Spiral Wave in Network of Neurons under Noise in.

[8] B.L. Hu, Jun Ma and J Tang: Plos One, (2013); 8(7): e69251, Selection of Multiarmed Spiral Waves in a Regular Network of Neurons.

[9] Jun Ma, Q.R. Liu and H.P. Ying: Commun Nonlinear Sci Numer Simulat, (2013); 18(7): 1665-1675, Emergence of Spiral Wave Induced by Defects Block. 
[10]Jun Ma, Y Wu and H.P. Ying: Chin Sci Bull, (2011); 56:151-7, Channel Noise-induced Phase Transition of Spiral Wave in Networks of Hodgkin-Huxley Neurons.

[11]Jun Ma, Jun Tang: Science China Technological Sciences, (2015): June, Transition of Electric Activity of Neurons Induced by Chemical and Electric Autapses.

[12]Jun Ma, Jian Xu: Published online, (2015)16November, An Introduction and Guidance for Neurodynamics.

[13]Jun Ma, Jun Tang: Science China Technological Sciences, (2015): December, A Review for Dynamics of Collective Behaviors of Network of Neurons.

[14]H.S. Chen, J.Q. Zhang and J.Q. Liu: Physica A, (2008); 87:1071-6, Enhancement of Neuronal Coherence by Diversity in Coupled Rulkov-map Models.

[15]Jun Ma, Jun Tang: Communications in Nonlinear Science and Numerical Simulation, Defects Formation, and Wave Emitting from Defects in Excitable Media.

[16] Vladimir: J Biol Syst, (2003); 11:57-84, Information Modelling of Neuronal Dynamics.

[17]Jun Ma, Wuyin Jin: Chaos Solitons \&fractals, Autapse-induced SynchrWonization in a Coupled Neuronal Network.

[18]Jun Ma, Jun Tang: EPL Europhysics Letters, Force Exerted on the Spiral Tip by the Heterogeneity in an Excitable Medium. 